\title{
ELECCIONES LEGISLATIVAS ESPAÑOLAS DE 6 DE JUNIO DE 1993
}

CARMEN FERNÁNDEZ-MIRANDA CAMPOAMOR

Profesora Titular de Derecho Constitucional 


\section{SUMARIO}

I. Campaña electoral.-II. Resultados electorales.-III. Valoración 


\title{
ELECCIONES LEGISLATIVAS ESPAÑOLAS DE 6 DE JUNIO DE 1993
}

\author{
POR \\ CARMEN FERNANDEZ-MIRANDA CAMPOAMOR \\ Profesora Titular de Derecho Constitucional
}

\section{Siglas de los principales partidos y coaliciones}

\begin{tabular}{|c|c|}
\hline 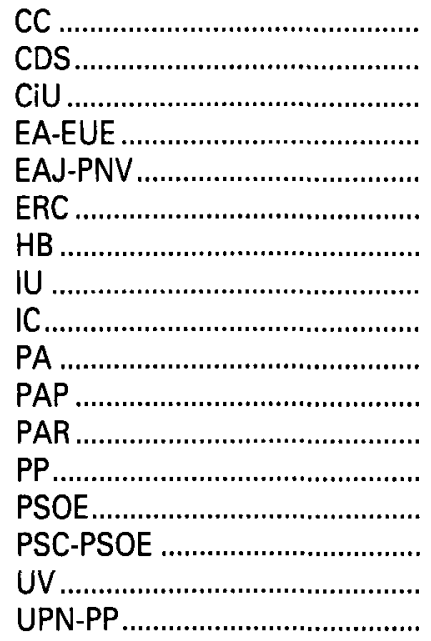 & $\begin{array}{l}\text { Coalición Canaria ' } \\
\text { Centro Democrático y Social } \\
\text { Convergéncia i Uniò } \\
\text { Eusko Alkartasuna-Euskal Ezquerra² } \\
\text { Eusko Alderdi Jestzalea (Partido Nacionalista Vasco) } \\
\text { Esquerra Republicana de Catalunya } \\
\text { Herri Batasuna } \\
\text { Izquierda Unida } \\
\text { Iniciativa per Catalunya } \\
\text { Partido Andalucista } \\
\text { Partido Andaluz del Progreso } \\
\text { Partido Aragonés Regionalista } \\
\text { Partido Popular } \\
\text { Partido Socialista Obrero Español } \\
\text { Partit dels Socialistes de Catalunya } \\
\text { Unión Valenciana } \\
\text { Unión del Pueblo Navarro-Partido Popular }\end{array}$ \\
\hline
\end{tabular}

1 Reciente coalición formada por partidos de distinta ideología: Iniciativa Canaria, Asamblea Majorera, Agrupación Independiente de Canarias, Centro Canario Independiente y Partido Nacionalista Canario.

${ }_{2}$ Euskal Ezquerra procede de la reciente escisión de Euskadiko Ezquerra, quien como tal partido - EE- se ha fusionado con el PSE-PSOE. 
El 12 de abril de 1993, el Presidente del Gobierno, Felipe González, disuelve las Cortes, de acuerdo con el art. 115 de la Constitución, convocando elecciones generales ${ }^{3}$ a celebrar el 6 de junio próximo. La causa de buscar la última decisión del electorado, pese a la mayoría importante ${ }^{4}$ de que goza el Partido Socialista en ambas Cámaras, radica en la existencia de una opinión pública muy crispada ante las continuas acusaciones parlamentarias de ineficacia gubernamental $y$, sobre todo, corrupción politica - muy aireada por los medios de comunicación-, y que afecta fundamentalmente a la financiación ilegal del Partido Socialista.

El 21 de mayo comienza la campaña electoral oficial ${ }^{5}$, animada por continuas encuestas que anuncian un cambio importante en la distribución de escaños en el Congreso de los Diputados.

Como caracteres generales de esta campaña, se pueden señalar los siguientes.

a) Alto índice de crispación y dureza; más que el debate sobre los problemas existentes y las posibles soluciones, lo que prima es la descalificación global del contrincante?

b) Enfrentamiento entre los líderes de los dos partidos mayoritarios - PP y PSOE - en un intento de captar el alto número de desencantados e indecisos en cuyas manos parece que está el peso del resultado, ante el virtual empate anunciado por los continuos sondeos publicados durante la campaña ${ }^{8}$. Para ello se utilizan, por ambas partes, instrumentos de dudoso gusto pero presunta eficacia: la relación entre el PP y la dictadura franquista - por parte de Felipe González-, poniendo en duda el mantenimiento de pensiones y subsidios, y dirigido directamente a la 3. ${ }^{\text {a }}$ edad, parados y pensionistas; y el "hay que echarlos" por ineficaces y corruptos, de José M.a Aznar, dirigido a la clase media agobiada por los impuestos.

3 RD 534/1993, de 12 de abril.

4176 diputados y 128 senadores; el Presidente del Gobierno justifica la disolución “...por el clima político crispado que impide afrontar la crisis económican.

5 La pre-campaña se había iniciado un mes antes: el 25 de abril, Felipe González en Badajoz y José M. a Aznar en Cáceres.

6 Como muestra, la macroencuesta elaborada por Demoscopia para El Pais (21 de marzo de 1993), de la que se publica un resumen en la pág. siguiente.

7 J. EstefaniA, "¿Quién gobernará el ajuste?», El País, 5-VI-93.

8 A. Ромво, "La decisión del voto indeciso", El Mundo, 5-VI-93. 
Voto válido y reparto de escaños ${ }^{6}$

\begin{tabular}{lcccccc}
\hline & \multicolumn{3}{c}{ Escaños } & \multicolumn{3}{c}{ Voto válido \% } \\
\cline { 2 - 8 } & Demoscopia & $\begin{array}{c}\text { CIS } \\
\text { noviembre } \\
1992\end{array}$ & $\begin{array}{c}\text { Elecciones } \\
\text { Generales } \\
1989\end{array}$ & Demoscopia & $\begin{array}{c}\text { CIS } \\
\text { noviembre } \\
1992\end{array}$ & $\begin{array}{c}\text { Elecciones } \\
\text { Generales } \\
1989\end{array}$ \\
\hline PSOE & $140-152$ & 155 & 175 & 33,9 & 36,3 & 39,6 \\
\hline PP & $137-149$ & 127 & 107 & 33,3 & 31,0 & 25,8 \\
\hline IU/IC & $26-29$ & 28 & 17 & 11,5 & 12,8 & 9,1 \\
\hline CDS & $0-1$ & 1 & 14 & 2,0 & 2,1 & 7,9 \\
\hline PNV & $5-6$ & 6 & 5 & 1,2 & 1,4 & 1,2 \\
\hline CiU & $16-20$ & 18 & 18 & 5,0 & 5,2 & 5,0 \\
\hline ERC & 1 & 1 & 0 & 0,8 & 0,7 & 0,4 \\
\hline HB & 4 & 4 & 4 & 0,9 & 1,1 & 1,1 \\
\hline EA & $1-2$ & 2 & 2 & 0,5 & 0,7 & 0,7 \\
\hline Verdes & $0-1$ & 0 & 0 & 2,8 & - & 1,6 \\
\hline AIC & 1 & 2 & 1 & 0,3 & 0,5 & 0,3 \\
\hline UV & 2 & 2 & 2 & 0,5 & 0,9 & 0,7 \\
\hline PAR & $0-1$ & 1 & 1 & 0,3 & 0,5 & 0,3 \\
\hline Otros & - & 3 & 4 & 4,8 & 4,8 & 5,6 \\
\hline Blanco & - & - & - & 2,0 & - & 0,7 \\
\hline & & & & & & \\
\hline
\end{tabular}

c) Se celebran, por primera vez en nuestra reciente democracia, debates televisados en las cadenas privadas, entre los dos principales líderes políticos ${ }^{9}$; la exclusión de representantes de otras opciones contribuye de forma clara a señalar el punto exacto del debate político, la posibilidad razonable de un cambio de tendencia en las urnas $y$, finalmente, la consolidación de un bipartidismo imperfecto ${ }^{10}$.

- La parcialidad de la TV pública en favor del partido del Gobierno fue denunciada en todos los medios de comunicación. Ver, por ejemplo, el editorial de $E$ I Pais de 5-VI-93.

${ }^{10}$ El Mundo, editorial de 6-VI-93; Diario 16; editorial de 5-VI-93. 
Si bien este medio novedoso se manifiesta mucho más eficaz que los mítines tradicionales, dirigidos a los incondicionales, es preciso resaltar la fugacidad del mensaje y la mayor influencia del último emitido; si en el primer encuentro el espectador presencia un Felipe González acorralado por las contundentes acusaciones - por parte de Aznar- de ineficacia, despilfarro, corrupción y arrogancia del partido gobernante, de lo que es responsable su secretario general; en el segundo debate, el líder socialista neutraliza las impresiones anteriores personalizando el mensaje ", obviando toda referencia al PSOE y resaltando su experiencia política $y$ los logros indudables de los 10 años de mandato, sobre todo su papel en la construcción europea; junto a ello, presenta a su contrincante como el representante de una derecha insolidaria con las economías más débiles, ante la que ha de unirse el voto progresista; frente a ello, José M. - Aznar se muestra incapaz de aportar algo convincente al panorama desolador que señala de forma demasiado reiterada. Si el debate es, en gran medida, dramatización, Felipe González es, sin duda, mejor actor.

d) En conclusión, finaliza la campaña, basada esencialmente en la denuncia ${ }^{12}$, con el convencimiento de que existe una razonable posibilidad de cambio político y que el alto número de indecisos va a determinar con su voto cuál es la opción ganadora ${ }^{13}$; el electorado mejor informado percibe cómo el punto de discusión no está en mantener las conquistas sociales presentes en todos los programas-, sino en cuál es la fórmula más eficaz para su financiación. En segundo lugar, existe la sensación generalizada de que acaba una etapa política: gane quien gane, no obtendrá los suficientes votos para gobernar sin el apoyo de otros partidos minoritarios; partidos que, a su vez, intentan resaltar su papel de posible apoyo parlamentario y su temor a ser inmolados ante el voto útil, tan imprescindible para los dos grandes.

Conviene señalar, para finalizar, el gran interés despertado en Europa por las elecciones españolas. Si bien Aznar busca el apoyo de los líderes políticos conservadores, aprovechando la tendencia hacia la derecha dominante en Europa, Felipe González, consciente del desprestigio de los partidos socialistas ${ }^{14}$, se enfrenta en solitario al electorado.

1 R. Cotarelo, "Lo dicho: ganará el PSOE", Diario 16, 5-VI-93.

12 Ver editorial de El Mundo y El País de 5-VI-93.

13 A. Pombo, "La decisión del voto indeciso", El Mundo, 5-VI-93.

14 Ver Le Point y Le Nouvel Observateur, semana 30 de mayo-6 de junio. No hay que olvidar la espectacular derrota del partido socialista francés en las elecciones generales de 21 de marzo de 1993; ello hace que los medios de comunicación del país vecino muestren una especial atención a las elecciones españolas. 


\section{RESULTADOS ELECTORALES}

\section{Resumen de los resultados electorales del Congreso de los Diputados ${ }^{15}$}

Electores

Votantes.

Votos válidos

Votos nulos

Votos en blanco

Participación
30.748 .763

23.718.051

23.590 .801

126.952

188.679

$77,29 \%$

\begin{tabular}{|c|c|c|c|c|}
\hline $\begin{array}{c}\text { Partidos, } \\
\text { federaciones } \\
y \text { coaliciones }\end{array}$ & $\begin{array}{c}\text { Votos } \\
\text { obtenidos } \\
\text { en } 1993\end{array}$ & Escaños & $\%$ aprox. & $\begin{array}{c}\text { Escaños } \\
\text { obtenidos } \\
\text { en } 1989\end{array}$ \\
\hline \multirow[t]{2}{*}{$\begin{array}{l}\text { PSOE } \\
\text { PSC-PSOE }\end{array}$} & $\begin{array}{l}7.872 .245 \\
1.277 .838\end{array}$ & $\begin{array}{r}141 \\
18\end{array}$ & 38,68 & \\
\hline & 9.150 .083 & 159 & & 175 \\
\hline \multirow[t]{2}{*}{$\begin{array}{l}\text { PP } \\
\text { UPN-PP }\end{array}$} & $\begin{array}{r}8.089 .235 \\
112.228\end{array}$ & $\begin{array}{r}138 \\
3\end{array}$ & 34,82 & \\
\hline & 8.201 .463 & 141 & & 107 \\
\hline $\mathrm{CiU}$ & 1.165 .783 & 17 & 4,96 & 18 \\
\hline \multirow[t]{2}{*}{$\begin{array}{l}\text { IU } \\
\text { IC }\end{array}$} & $\begin{array}{r}1.905 .673 \\
273.444 \\
\end{array}$ & $\begin{array}{r}15 \\
3 \\
\end{array}$ & 9,57 & \\
\hline & 2.179 .117 & 18 & & 17 \\
\hline EAJ-PNV & 291.448 & 5 & 1,24 & 5 \\
\hline $\mathrm{CC}^{16}$ & 207.077 & 4 & 0,88 & 4 \\
\hline $\mathrm{HB}$ & 206.876 & 2 & 0,88 & \\
\hline ERC & 189.632 & 1 & 0,80 & \\
\hline PAR & 144.544 & 1 & 0,61 & 1 \\
\hline EA-EUE & 129.293 & 1 & 0,55 & 2 \\
\hline
\end{tabular}

15 Ver ampliados en "B.O.E." n. 169, de 16 de julio de 1993.

16 CC incluye a AIC (Agrupación Independiente de Canarias), que obtuvo un escaño en 1989. 


\section{Resumen de los resultados electorales del Congreso de los Diputados (continuación)}

\begin{tabular}{lcccc}
\hline $\begin{array}{c}\text { Partidos, } \\
\text { federaciones } \\
\text { y coaliciones }\end{array}$ & $\begin{array}{c}\text { Votos } \\
\text { obtenidos } \\
\text { en } 1993\end{array}$ & Escaños & \% aprox. & $\begin{array}{c}\text { Escaños } \\
\text { obtenidos } \\
\text { en 1989 }\end{array}$ \\
\hline UV & 112.341 & 1 & 0,48 & 2 \\
\hline CDS & 414.740 & 0 & & 14 \\
\hline PA & 96.513 & 0 & & 2 \\
\hline AIC & & & 1 \\
\hline EE $^{17}$ & & & 2 \\
\hline
\end{tabular}

Resumen de los resultados electorales en el Senado ${ }^{18}$

\begin{tabular}{lcccr}
\hline Partidos & Electos & Desig. autonómica & Total 1993 & Total 1989 \\
\hline PSOE & 96 & 21 & 117 & $129(108+21)$ \\
\hline PP & 93 & 14 & 107 & $91(77+14)$ \\
\hline CiU & 10 & 5 & 15 & $14(10+4)$ \\
\hline PNV & 3 & 2 & 5 & $6(4+2)$ \\
\hline CC & 5 & 1 & 6 & - \\
\hline UU & 0 & 2 & 2 & $3(1+2)$ \\
\hline PAR & 0 & 1 & 1 & \\
\hline EA & 0 & 1 & 1 & \\
\hline UV & 0 & 1 & 1 & \\
\hline HB & 1 & - & 1 & $3(3+0)$ \\
\hline CDS & 0 & - & 0 & $1(1+0)$ \\
\hline Otros & 0 & - & 0 & $8(4+4)$ \\
\hline TotAL & 208 & 48 & 256 & 255 \\
\hline & & & &
\end{tabular}

17 EE (Euskadiko Ezquerra), fusionado actualmente con el PSOE, obtuvo dos escaños en 1989.

18 Igualmente, ampliación en el "B.O.E." n. 169, de 16 de julio de 1993, con los votos obtenidos por cada Senador. Hay que tener en cuenta que el Senado está formado por un Senador más que en 1989, de designación autonómica por Barcelona, y corresponde a CiU. La participación en las elecciones al Senado fue más alta que al Congreso: $77,37 \%$. 
Resultados electorales al Congreso de los Diputados por CC.AA.

ANDALUCÍA: 61 Diputados.

Triunfo del PSOE en todas las provincias; el PP es la segunda fuerza en todas las provincias. Desaparición del PA como fuerza parlamentaria. IU logra 4 escaños (Sevilla, Córdoba, Málaga, Cádiz).

\begin{tabular}{|c|c|c|}
\hline & 1993 & 1989 \\
\hline $\begin{array}{l}\text { PSOE } \\
\text { PP } \\
\text { IU } \\
\text { PA }\end{array}$ & $\begin{array}{r}37 \\
20 \\
4 \\
0\end{array}$ & $\begin{array}{r}42 \\
12 \\
5 \\
2\end{array}$ \\
\hline
\end{tabular}

ARAGÓN: 13 Diputados.

Triunfo del PSOE en todas las provincias; el PP es la segunda fuerza en todas las provincias; el PAR e IU mantienen un escaño en Zaragoza.

\begin{tabular}{|c|c|c|}
\hline & 1993 & 1989 \\
\hline PSOE & 7 & 7 \\
\hline PP & 4 & 4 \\
\hline PAR & 1 & 1 \\
\hline 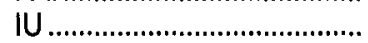 & 1 & $i$ \\
\hline
\end{tabular}

ASTURIAS: 9 Diputados.

Empate en n. de escaños entre PSOE y PP; más votado el primero. Desaparece el CDS como fuerza parlamentaria.

\begin{tabular}{|c|c|c|}
\hline & 1993 & 1989 \\
\hline 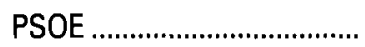 & 4 & 4 \\
\hline PP & 4 & 3 \\
\hline 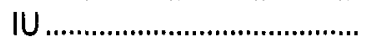 & 1 & 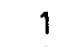 \\
\hline CDS & 0 & 1 \\
\hline
\end{tabular}


EXTREMADURA: 11 Diputados.

Triunfo del PSOE en ambas provincias.

\begin{tabular}{|c|c|c|}
\hline & 1993 & 1989 \\
\hline . & 7 & 7 \\
\hline PP & 4 & 4 \\
\hline
\end{tabular}

GALICIA: 26 Diputados; pierde uno por razones demográficas.

Triunfo del PP en todas las provincias, salvo Orense, en que logra igual número de escaños que el PSOE, aunque obteniendo más votos.Desaparece el CDS (La Coruña) como fuerza parlamentaria.

\begin{tabular}{|c|c|c|}
\hline & 1993 & 1989 \\
\hline 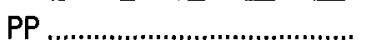 & 15 & 14 \\
\hline 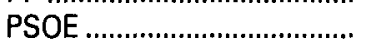 & 11 & 12 \\
\hline CDS & 0 & 1 \\
\hline
\end{tabular}

CASTILLA Y LEÓN: 33 Diputados.

Triunfo del PP en todas las provincias. EI PSOE pierde la supremacia en Valladolid y León y el empate con el PP en Salamanca, Burgos y Ávila. Desaparece el CDS (Ávila) como fuerza parlamentaria.

\begin{tabular}{lrr}
\hline & 1993 & 1989 \\
\hline PP $\ldots \ldots \ldots \ldots \ldots \ldots \ldots \ldots \ldots \ldots \ldots \ldots \ldots \ldots \ldots \ldots \ldots \ldots \ldots \ldots \ldots$ & 20 & 17 \\
PSOE $\ldots \ldots \ldots \ldots \ldots \ldots \ldots \ldots \ldots \ldots \ldots \ldots \ldots \ldots \ldots \ldots$ & 13 & 15 \\
CDS $\ldots \ldots \ldots \ldots \ldots \ldots \ldots \ldots \ldots \ldots \ldots \ldots \ldots$ & 0 & 1 \\
\hline
\end{tabular}

CASTILLA-LA MANCHA: 20 Diputados.

Triunfo del PSOE en Toledo y Ciudad Real; triunfo del PP en Cuenca y Guadalajara; empate en $n .^{\circ}$ de escaños en Albacete, aunque es más votado el PSOE.

\begin{tabular}{|c|c|c|}
\hline & 1993 & 1989 \\
\hline $\begin{array}{l}\text { PSOE } \\
\text { PP }\end{array}$ & $\begin{array}{l}10 \\
10\end{array}$ & \\
\hline
\end{tabular}


BALEARES: 7 Diputados (uno más que en 1989, por razones demográficas).

Triunfo del PP.

\begin{tabular}{lrr}
\hline & 1993 & 1989 \\
\hline PP $\ldots \ldots \ldots \ldots \ldots \ldots \ldots \ldots \ldots \ldots \ldots \ldots \ldots \ldots \ldots \ldots \ldots \ldots \ldots \ldots \ldots \ldots \ldots \ldots \ldots \ldots \ldots$ & 4 & 3 \\
PSOE & 3 & 3 \\
\hline
\end{tabular}

CATALUÑA: 47 Diputados (uno más, por razones demográficas: Tarragona)

Triunfo del PSC-PSOE, que gana en Tarragona y Barcelona; CIU gana en Girona y Lleida. EI CDS desaparece y ERC logra un escaño en Barcelona. IU, aunque mantiene el mismo número de escaños, es más votado que en 1989.

\begin{tabular}{|c|c|c|}
\hline & 1993 & 1985 \\
\hline PSC-PSOE $\ldots \ldots \ldots \ldots \ldots \ldots \ldots$ & 18 & 20 \\
\hline CIU & 17 & 18 \\
\hline PP & 8 & 4 \\
\hline IU & 3 & 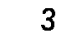 \\
\hline ERC & 1 & 1 \\
\hline CS & 0 & a \\
\hline
\end{tabular}

MADRID: 34 Diputados (uno más por razones demográficas).

Triunfo del PP. EI CDS desaparece como fuerza parlamentaria.

\begin{tabular}{|c|c|c|}
\hline & 1993 & 1989 \\
\hline ................... & 16 & 12 \\
\hline PSOE ................................. & 13 & 12 \\
\hline IU & 5 & 5 \\
\hline CDS …............................... & 0 & 4 \\
\hline
\end{tabular}


PAÍS VASCO: 19 Diputados; pierde dos por razones demográficas.

Triunfo del PSOE; gana en Álava y Guipúzcoa; empata en Vizcaya en n.. de escaños con el PNV, siendo más votado este último ${ }^{19}$. HB sólo logra dos escaños (Vizcaya y Guipúzcoa, perdiendo el predominio en ésta última). EA sólo consigue un Diputado en Guipúzcoa.

\begin{tabular}{|c|c|c|}
\hline & 1993 & 1989 \\
\hline PSOE ............ & 7 & 6 \\
\hline 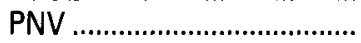 & 5 & 5 \\
\hline PP & 4 & 2 \\
\hline 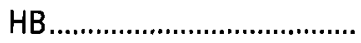 & 2 & 4 \\
\hline EA & 1 & 2 \\
\hline 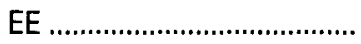 & - & 2 \\
\hline
\end{tabular}

NAVARRA: 5 Diputados.

Triunfo de UPN-PP.

\begin{tabular}{rrr}
\hline & 1993 & 1989 \\
\hline UPN-PP ................................ & 3 & 3 \\
PSOE ………………….... & 2 & 2 \\
\hline
\end{tabular}

COMUNIDAD VALENCIANA: 31 Diputados.

Triunfo del PP en todas las provincias, con lo que el PSOE pierde la tradicional hegemonia. UV sólo logra un Diputado en Valencia. Desaparece el CDS como fuerza parlamentaria e IU logra un importante ascenso.

\begin{tabular}{|c|c|c|}
\hline & 1993 & 1989 \\
\hline & 15 & 9 \\
\hline PSOE & 12 & 16 \\
\hline 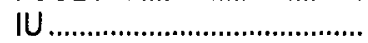 & 3 & , \\
\hline$\ldots$ & 1 & 2 \\
\hline CDS & 0 & 1 \\
\hline
\end{tabular}

19 Hay que recordar que $E E$, con dos escaños en 1989, se ha fusionado con el PSE-PSOE. 
CANARIAS: 14 Diputados.

Triunfo del PSOE en Tenerife y del PP en Gran Canaria; más votado el PP. El CDS desaparece como fuerza parlamentaria. EI CC, de reciente aparición, logra cuatro escaños ${ }^{20}$.

\begin{tabular}{|c|c|c|}
\hline & 1993 & 1989 \\
\hline ......... & 5 & 3 \\
\hline PSOE. & 5 & 7 \\
\hline $\mathrm{CC}$ & 4 & - \\
\hline CDS & 0 & 3 \\
\hline AIC & - & 1 \\
\hline
\end{tabular}

CANTABRIA: 5 Diputados.

Triunfo del PSOE.

\begin{tabular}{|c|c|c|}
\hline & 1993 & 1989 \\
\hline PSOE & $\begin{array}{l}3 \\
2\end{array}$ & $\begin{array}{l}3 \\
2\end{array}$ \\
\hline
\end{tabular}

LA RIOJA: 4 Diputados.

Empate en $n .{ }^{\circ}$ de escaños entre PP y PSOE, siendo más votado el primero.

\begin{tabular}{|c|c|c|}
\hline & 1993 & 1989 \\
\hline PP & $\begin{array}{l}2 \\
2\end{array}$ & $\begin{array}{l}2 \\
2\end{array}$ \\
\hline
\end{tabular}

${ }^{20}$ Coalición formada por los siguientes partidos: Iniciativa Canaria, Asamblea Majorera, Agrupación independiente de Canarias, Centro Canario Independiente y Partido Nacionalista Canario. 
MURCIA: 9 Diputados.

Empate en $n .^{\circ}$ de escaños entre PP y PSOE, siendo más votado el $P P$, que gana al PSOE por vez primera. IU logra un escaño y desaparece el CDS.

\begin{tabular}{|c|c|c|}
\hline & 1993 & 1989 \\
\hline$\ldots$ & 4 & 3 \\
\hline PSOE & 4 & 5 \\
\hline 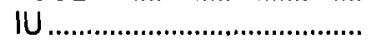 & 1 & 0 \\
\hline CDS & 0 & 1 \\
\hline
\end{tabular}

CEUTA: 1 Diputado, que logra el PP.

\begin{tabular}{|c|c|c|}
\hline & 1993 & 1989 \\
\hline $\begin{array}{l}\text { PP ............................................... } \\
\text { PSOF }\end{array}$ & $\begin{array}{l}1 \\
0\end{array}$ & 1 \\
\hline
\end{tabular}

MELILLA: 1 Diputado, que logra el PSOE.

\begin{tabular}{|c|c|c|}
\hline & 1993 & 1989 \\
\hline $\begin{array}{l}\text { PSOE } \\
\text { PP }\end{array}$ & $\begin{array}{l}1 \\
0\end{array}$ & $\begin{array}{l}0 \\
1\end{array}$ \\
\hline
\end{tabular}

En ambas zonas se invierte el voto respecto a 1989. 
Resultados electorales en el Senado por CC.AA.

ANDALUCÍA: 32 Senadores.

PSOE ............. 24

PP ................ 8

ARAGÓN: 12 Senadores.

PSOE ............. 9

PP ................ 3

ASTURIAS: 4 Senadores.

PSOE ............. 3

PP ................. 1

EXTREMADURA: 8 Senadores.

PSOE ............. 6

PP ................ 2

GALICIA: 16 Senadores.

PP ................ 12

PSOE ............. 4

CASTILLA Y LEÓN: 36 Senadores.

PP ….............. 27

PSOE ............ 9 
CASTILLA-LA MANCHA: 20 Senadores.

$\begin{array}{lr}\text { PSOE } \ldots \ldots \ldots \ldots . . . & 11 \\ \text { PP ................... } & 9\end{array}$

BALEARES: 5 Senadores.

PP …............. 4

PSOE ............. 1

CATALUÑA: 16 Senadores.

CiU ................ 10

PSOE ............. 6

MADRID: 4 Senadores.

PP …............. 3

PSOE ............. 1

PAIS VASCO: 12 Senadores.

$\begin{array}{lr}\text { PSOE ............. } & 7 \\ \text { PNV ............... } & 3 \\ \text { PP ................. } & 1 \\ \text { HB ................. } & 1\end{array}$

NAVARRA: 4 Senadores.

UPN-PP ........ 3

PSOE ............ 1

COMUNIDAD VALENCIANA: 12 Senadores.

PP .................. 8

PSOE ............. 4 
CANARIAS: 11 Senadores.

$\begin{array}{lr}\text { CC ................ } & 5 \\ \text { PSOE …............ } & 4 \\ \text { PP ................. } & 2\end{array}$

CANTABRIA: 4 Senadores.

PSOE ............ 3

PP ................ 1

LA RIOJA: 4 Senadores.

PP …............. 3

PSOE ............. 1

MURCIA: 4 Senadores

PP ................ 3

PSOE ............. 1

CEUTA: 2 Senadores.

PP ................ 2

MELILLA: 2 Senadores.

$\begin{array}{ll}\text { PSOE ............... } 1 & 1 \\ \text { PP } & 1\end{array}$ 
En conclusión, y respecto al Congreso de los Diputados, se puede señalar lo siguiente.

EI PSOE gana las elecciones aunque pierde 16 escaños $y$, en consecuencia, la mayoría holgada de que disfrutaba. EI PP, por el contrario, asciende de forma sobresaliente con un aumento de 34 escaños $y$, de momento, una barrera difícil de superar en cuatro Comunidades: Extremadura, Andalucía, País Vasco y Cataluña; pese a lo dicho, en las dos últimas ha dejado de ser un partido marginal para convertirse en una opción capaz de competir con los nacionalismos.

EI PSOE triunfa en: Andalucía, Extremadura, Cantabria, País Vasco, Cataluña, Aragón y Melilla. EI PP, a su vez, en Castilla y León, Galicia, Madrid, Baleares, Valencia, Navarra y Ceuta. Ambos empatan en número de escaños en Asturias (con más votos del PSOE) y en Castilla-La Mancha, Canarias, Murcia y La Rioja (con más votos del PP).

IU logra un escaño más que en 1989; mantienen el mismo número el PNV (disminuyendo votos) y el PAR (aumentando votos). Pierden escaños CiU, EA, UV (-1) y HB (-2). Desaparece del hemiciclo el CDS (-14) $y$ el PA (-2).

Respecto al Senado, es igualmente el PSOE el partido más votado, aunque pierde la mayoría absoluta y 12 Senadores; el PP avanza de forma importante, logrando 16 escaños más que en 1989. EI PSOE triunfa en Andalucía, Extremadura, Cantabria, País Vasco, Aragón, Asturias y Castilla-La Mancha. EI PP, a su vez, en Galicia, Castilla y León, Baleares, Madrid, Navarra, Comunidad Valenciana, La Rioja, Murcia y Ceuta. Empatan PP y PSOE en Melilla, siendo más votado el segundo. CiU vence en Cataluña y $\mathrm{CC}$ en Canarias; hay que resaltar el pobre resultado nacionalista y regionalista en la Cámara que se dice de representación territorial.

EI CDS pierde el único escaño que habia obtenido en 1989, convirtiéndose en partido extraparlamentario; igualmente IU pierde el escaño logrado en la convocatoria anterior, aunque tiene en la Cámara dos Senadores de designación autonómica. 


\section{VALORACIÓN}

Observados los resultados electorales, se pueden valorar de la siguiente forma, teniendo en cuenta que la confrontación de los dos grandes partidos estatales va a ser determinante.

\section{A) Valoración general}

1. Triunfo, por cuarta vez consecutiva, del PSOE como consecuencia de la alta participación electoral, generada por una campaña en que se presenta el voto ciudadano como esencial para mantener una opción política o dar entrada a otra alternativa; el voto de buena parte de la abstención proveniente del PSOE, junto a presuntos votantes de IU y del CDS, van a dar la victoria a la opción de Felipe González. El último debate televisivo es causa directa de los resultados.

2. Pese a esta victoria, el PSOE sufre una importante disminución de escaños, que le privan de la mayoría absoluta y cuasi-absoluta que venía disfrutando desde 1982.

3. Importante ascenso del PP ${ }^{22}$, quien, pese a los pronósticos de todos los medios de comunicación y al propio ánimo triunfalista de sus militantes, no logra superar al partido socialista, aunque sí convertirse en una alternativa cierta de gobierno.

4. Estancamiento del nacionalismo y regionalismo salvo $\mathrm{CC}$ en beneficio de los partidos estatales, como respuesta del electorado a la dura confrontación entre éstos. Igual causa lleva al exiguo crecimiento de IU, también en contra de los sondeos de opinión, y a la desaparición del CDS; muchos de sus presuntos votantes prefirieron, en el último momento, votar socialista o popular.

5. El Parlamento salido de las urnas se caracteriza por un bipartidismo imperfecto en que, junto a dos grandes opciones políti-

22 Ver editorial de Diario 16 de 8-VI-93 y de El Pais de 7-VI-93. 
cas que ocupan el $87,71 \%{ }^{23}$ del hemiciclo, existen otras opciones minoritarias capaces de posibilitar pactos de gobierno. La pérdida de la mayoría holgada de que disfrutaba el PSOE da entrada a la necesidad continua de negociación y consenso, iniciándose un nuevo lenguaje político.

6. Pese al importante avance del centro-derecha, consecuencia del rechazo ciudadano al desprestigio socialista, sigue dominando la opción de izquierda, a diferencia de lo ocurrido en el resto de los países comunitarios ${ }^{24}$; hay que tener en cuenta el dominio socialista en zonas tan amplias como Extremadura y Andalucía, así como la menor implantación del PP pese a su ascenso en el País Vasco y Cataluña ${ }^{25}$. Sin embargo, se constata que el voto urbano y el voto joven, en general, se decantan hacia el PP.

Porcentajes de votación en las ciudades más pobladas (Resultados del Ministerio del Interior - "El Pais", 15-VI-93) *

\begin{tabular}{|c|c|c|c|c|c|}
\hline \multirow[b]{2}{*}{ Más de 500.000 habitantes } & \multirow[t]{2}{*}{$P P$} & \multirow[t]{2}{*}{$P S O E$} & \multirow[t]{2}{*}{ U } & \multicolumn{2}{|c|}{$\begin{array}{l}\text { Nacionalistas } \\
\text { Regionalistas }\end{array}$} \\
\hline & & & & & \\
\hline 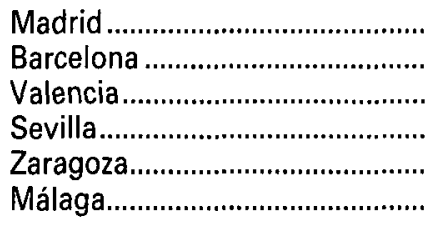 & $\begin{array}{r}47,7 \\
21,7 \\
42,6 \\
32,7 \\
32,8 \\
35,0\end{array}$ & $\begin{array}{r}32,0 \\
28,9 \\
31,1 \\
49,0 \\
29,2 \\
41,6\end{array}$ & $\begin{array}{r}13,7 \\
8,1 \\
12,5 \\
11,0 \\
13,1 \\
16,7\end{array}$ & $\begin{array}{r}32,5 \\
8,1 \\
3,4 \\
20,4 \\
1,5\end{array}$ & $\begin{array}{l}\text { CiU } \\
\text { UV } \\
\text { PA } \\
\text { PAR } \\
\text { PA }\end{array}$ \\
\hline \multicolumn{6}{|l|}{ De 300.000 a 500.000 habitantes } \\
\hline 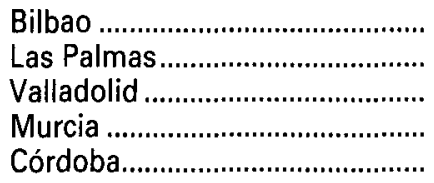 & $\begin{array}{r}20,9 \\
43,4 \\
44,7 \\
53,0 \\
34,7\end{array}$ & $\begin{array}{r}23,7 \\
22,4 \\
34,0 \\
32,8 \\
\mathbf{3 6 , 7}\end{array}$ & $\begin{array}{r}6,8 \\
7,0 \\
14,0 \\
9,8 \\
22,7\end{array}$ & $\begin{array}{r}28,5 \\
16,5\end{array}$ & $\begin{array}{l}\text { PNV } \\
\text { CC }\end{array}$ \\
\hline
\end{tabular}

23 Antes el $80,57 \%$.

${ }^{24}$ Ver J. PRIETO, "El voto sigue instalado en el centro izquierda", El País, 8VI-93.

25 G. Jackson, "Interpretación de las elecciones", en El País, 12-VI-93.

* En negrita, el partido más votado. 
Porcentajes de votación en las ciudades más pobladas (continuación) (Resultados del Ministerio del Interior - "El Pais», 15-VI-93) *

\begin{tabular}{|c|c|c|c|c|}
\hline & $P P$ & PSOE & $\mathbb{N}$ & $\begin{array}{l}\text { Nacionalistas } \\
\text { Regionalistas }\end{array}$ \\
\hline \multicolumn{5}{|l|}{ De 200.000 a 300.000 habitantes } \\
\hline 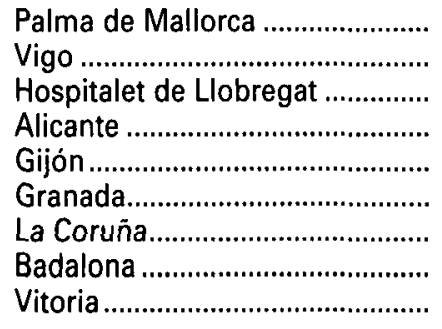 & $\begin{array}{r}44,9 \\
46,4 \\
16,4 \\
45,3 \\
37,7 \\
45,8 \\
41,9 \\
14,8 \\
20,1\end{array}$ & $\begin{array}{r}34,2 \\
30,6 \\
47,8 \\
36,9 \\
36,2 \\
35,9 \\
37,2 \\
46,3 \\
28,1\end{array}$ & $\begin{array}{r}7,7 \\
10,0 \\
11,7 \\
11,9 \\
17,2 \\
12,0 \\
7,7 \\
10,5 \\
12,1\end{array}$ & $\begin{aligned} 0,9 & \text { PA } \\
7,6 & \text { BNG } \\
21,1 & \text { CiU } \\
13,1 & \text { PNV }\end{aligned}$ \\
\hline \multicolumn{5}{|l|}{ De 160.000 a 200.000 habitantes } \\
\hline 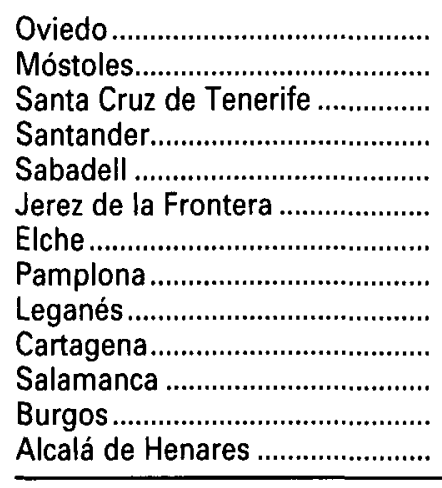 & $\begin{array}{r}47,0 \\
35,1 \\
34,0 \\
39,3 \\
12,4 \\
25,0 \\
39,7 \\
37,5 \\
27,6 \\
46,5 \\
50,6 \\
50,1 \\
34,7 \\
\end{array}$ & $\begin{array}{r}32,3 \\
40,1 \\
25,3 \\
35,3 \\
43,1 \\
47,8 \\
44,7 \\
30,0 \\
45,1 \\
36,1 \\
32,4 \\
32,4 \\
40,5 \\
\end{array}$ & $\begin{array}{r}12,4 \\
16,8 \\
6,2 \\
8,7 \\
9,8 \\
8,9 \\
9,5 \\
10,3 \\
19,7 \\
12,3 \\
9,0 \\
9,0 \\
17,5 \\
\end{array}$ & $\begin{array}{rl}30,0 & \mathrm{CC} \\
6,4 & \text { UPCA } \\
27,6 & \mathrm{CiU} \\
13,8 & \mathrm{PAP} \\
11,6 & \mathrm{HB}\end{array}$ \\
\hline
\end{tabular}

\section{B) Valoración por partidos}

1. Sorprendente el triunfo del PSOE ${ }^{26}$, pese a la alta tasa de desempleo, la profunda crisis económica y el acoso de los medios de comunicación desvelando continuos escándalos de corrup-

* En negrita, el partido más votado.

${ }^{26}$ Claro fallo de los sondeos debido a que sus pronósticos se fundamentaban en el alto nivel de crispación politica producido por el desastre del último mandato de Felipe González. Ver editorial de Diario 16, 7-VI-93. 
ción; incluso frente a las previsiones dadas por los sondeos sobre una intención de voto partidaria de un cambio político. Es Felipe González, obviando al partido socialista, quien logra un difícil voto de confianza del electorado más crítico, en respuesta a una falaz y eficaz dicotomía: o yo o la derecha franquista; es decir, el equilibrado Presidente del Gobierno ha usado los argumentos propios del sector guerrista $y$ ha movilizado a los votantes incondicionales y a aquellos otros escépticos que le apoyarán por temor a un paso atrás ${ }^{27}$. Son dignos de resaltar dos aspectos. En primer lugar, la eficacia de ese mensaje del miedo que demuestra un subsistente recelo ante la derecha tradicional española, que la opción de Aznar aún no ha podido superar. En segundo lugar, el PSOE con su triunfo va a heredar, sin embargo, una complicada situación económica interior, de la que es responsable, aunque inscrita en la global de Europa, que afecta directamente a todos los miembros de la Unión; es, pues, una victoria con doble faz.

2. EI PP logra un importante ascenso, consecuencia del desprestigio del partido socialista, manejado de forma catastrofista por Aznar; sin embargo, no logra superar a aquél. Resulta más claro su mensaje negativo de crítica sin concesiones, que el positivo de aportar soluciones $y$ ofrecer eficacia y honradez; por eso, el electorado en situación de inclinar la balanza hacia el PP, se sentirá más movido por la advertencia de Felipe González sobre la derecha insolidaria capaz de recortar las libertades democráticas y las cotas de bienestar.

Pese a todo, el PP se ha hecho mayoritariamente con el voto urbano y el voto joven; sin embargo, Andalucía, Extremadura y los cinturones industriales de las grandes ciudades, unido al pobre resultado en el País Vasco y Cataluña, pese a lograr implantarse donde ha de competir con opciones nacionalistas de ideologia similar, son claves para entender el fracaso, dentro de su éxito, de la opción de Aznar.

27 M. SUÁREZ, "El tándem ha vuelto a funcionar», en Diario 16 de 8-VI-93. J. NavarRo, "Felipe cabalga de nuevo", en El Mundo de 7-VI-93. R. DEL Pozo, "Dia a día, hora a horan, en Diario 16 de 9-VI-93.

28 Ver editorial de Diario 16 de 11-VI-93.

29 F. Sahagún, "La nueva España de Felipe González", El Mundo 9-VI-93. A. Delgado-GaL, "Después de la resaca", Diario 16, 13-VI-93. P. J. RamireZ, "La factura de la historia", El Mundo, 13-VI-93. J. LACALLE, "Aznar y el pasado de la derecha", Diario 16, 8-VI-93. 
3. IU avanza muy poco, pese a las expectativas despertadas por los sondeos ${ }^{30}$; obtiene solamente un Diputado más que en 1989 y, en el Senado, pierde el representante que había logrado. Como causas de estos resultados, creo que independientes de la enfermedad de Anguita, pueden señalarse las siguientes. En primer lugar, el dominio del aparato del PCE en la coalición, no situando len las listas electorales a los renovadores de más prestigio ${ }^{31}$, capaces de dar un sesgo a la izquierda y superar el hundimiento del comunismo. En segundo lugar, al presunto votante de IU le movió más el miedo al triunfo de la derecha que el mensaje un tanto utópico del líder de la coalición; es decir, no creyó que votando a IU pudiera evitar el triunfo de Aznar y, en consecuencia, apoyó a Felipe González, quien logró concentrar mayoritariamente los votos de la izquierda.

4. Desaparición anunciada del CDS, consecuencia del enfrentamiento entre dos grandes partidos, situados en posiciones políticas de centro; los presuntos votantes del CDS se han visto obligados a respaldar una de las dos opciones para evitar el triunfo de la otra: el voto útil ha acabado con el partido de Calvo Ortega ${ }^{32}$.

5. Desde el inicio de la campaña se señala el papel importante que pueden tener nacionalismos y regionalismos ante el mapa electoral que se pronostica; se consideran las opciones que van a permitir los pactos necesarios para hacer posible la estabilidad política. Sin embargo, y a la vista de los resultados electorales, estos partidos no han crecido ${ }^{33}$; si acaso, se han mantenido en su conjunto, cediendo votos a las opciones estatales como consecuencia nuevamente del enfrentamiento entre PSOE y PP. En Cataluña aumenta de forma importante la fuerza del PP en menoscabo de CiU, quien, a su vez, es vencida por el PSOE, no logrando ser, como pretendía, la represen-

${ }^{30}$ R. G. Díez, "Izquierda Unida, sí, pero", Diario 16;8-VI-93. R. CAStRo, "Izquierda Unida, la gran decepción", El Mundo, 7-VI-93.

${ }^{31}$ Los lideres de Nueva Izquierda, N. Sartorius, C. Almeida y P. Castellanos. Hay que tener en cuenta que entre los Diputados elegidos hay mayor número de renovadores (10) que de comunistas clásicos (8); sólo se lograron superar los escaños obtenidos en 1989 en Murcia (de 0 a 1) y en Valencia (de 2 a 3), donde domina el sector renovador.

32 J. Morales, "CDS o el canto del cisne", Diario 16, 9-VI-93.

33 X. Horcajo, "¿Ahora decidirá CiU?", Diario 16, 8-VI-93. M. Onaindía, "Elecciones sin Artapalo", Diario 16, 9-VI-93. A. HERCE, "Recuperar la hegemonía perdida" y "El revés electoral", Diario 16, 9-VI-93. 
tación catalana mayoritaria en el Parlamento del Estado; pierde un escaño en el Congreso y se mantiene en el Senado. El partido independentista ERC logra un representante en el Congreso.

En el País Vasco HB sufre una importante pérdida de votos y su dominio en Guipúzcoa, tras el descenso en el apoyo sociológico a ETA. EI PNV se estanca y puede afirmarse que en esta C.A. existe, por vez primera, la misma dinámica que en el resto del Estado; es decir, apoyo al PSOE o al PP y, en consecuencia, aumento de votos del primero, que se beneficia de su fusión con $E E$, y del segundo, que dobla el número de escaños logrado en 1989. EI PNV compensa el apoyo logrado por el partido de Aznar con votos presuntamente destinados a EA, que pierde un Diputado.

Respecto de las opciones minoritarias representadas en el Parlamento elegido en 1989 PAR, UV, EA y PS sufren pérdida de votos y de escaños; la razón vuelve a ser la necesidad de un apoyo frontal a las dos opciones estatales mayoritarias, con lo que, según su ideología, los votos van a una o a otra. En el caso del PA, su propia crisis interna y la división originada por el reciente PAP de P. Pacheco, le perjudica notablemente, perdiendo los dos Diputados que tenía y convirtiéndose en partido extraparlamentario. La única excepción al estancamiento nacionalista es la Coalición Canaria, que logra unir los votos críticos a la gestión del PSOE en las Islas por encima de las ideologías ${ }^{34}$; consigue una representación de 4 Diputados en el Congreso y de 5 Senadores; es decir, voz propia del nacionalismo canario y capacidad de pacto político ${ }^{35}$.

\section{C) La participación electoral}

Las elecciones se celebran con una participación superior al $77 \%$; es decir, casi seis puntos por encima de la de 1989 y sólo comparable a la registrada en momentos de especial relevancia política, tales como 1977 y 1982. Ello es debido, en primer lugar, al interés del electorado an-

34 Reducir el alto índice de paro y lograr una nueva Ley del Régimen Económico y Fiscal de Canarias, son las principales pretensiones del nuevo grupo parlamentario canario.

${ }^{35}$ C. PeñAte, "El auge del nacionalismo isleño", Diario 16, 9-VI-93. 
te la posibilidad de un cambio político; en segundo lugar, al empate anunciado por los sondeos de intención de voto, empate que motiva al abstencionista habitual a acudir a las urnas; al ciudadano que sólo vota cuando entiende que es imprescindible el apoyo a la opción política que menos le desagrada. Las CC.AA. con mayor participación fueron Valencia $(82,25 \%)$, Murcia $(81,85 \%)$, Extremadura $(80,65 \%)$ y Cantabria $(80,17 \%)$; las de menos, País Vasco $(69,71 \%)$ y Galicia $(70,82 \%)$.

Participación-abstención en elecciones legislativas ${ }^{36}$

\begin{tabular}{|c|c|c|c|}
\hline Años & Abstención & Participación & \\
\hline 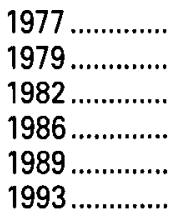 & 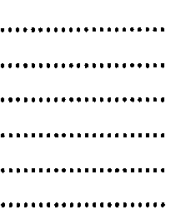 & $\begin{array}{l}21,43 \\
31,31 \\
20,13 \\
29,60 \\
30,27 \\
22,71\end{array}$ & $\begin{array}{l}78,57 \\
68,09 \\
79,87 \\
70,40 \\
69,73 \\
77,29\end{array}$ \\
\hline
\end{tabular}

Tomando como referencia la abstención producida en las anteriores elecciones legislativas $-30,27 \%$ - respecto a la actual $-22,71 \%-$ y el destino del voto joven recién incorporado a la participación electoral, se pueden señalar los siguientes datos ${ }^{37}$.

En 1989 se produce una abstención de 2.719 .000 votantes, de los que en 1993:

1.600 .000 votan al PSOE

1.023.000 votan al PP

53.000 votan a IU.

Respecto a 1.580 .000 jóvenes que votan por vez primera:

545.000 lo hacen al PP

471.000 lo hacen al PSOE

209.000 lo hacen a IU

15.000 lo hacen al CDS.

36 Fuente: Ministerio del Interior.

${ }^{31}$ Según un estudio realizado por C. Malo de Molina, J. M. de Elias y C. Prieto Valentin-Gamazo, a partir de la base documental de Sigma-dos (6-VI-93), publicado por El Mundo, 13-VI-93, del que extraemos un resumen. 
Es decir, si la mayor participación beneficia claramente al PSOE, los jóvenes se decantan mayoritariamente por el PP. Sin embargo, uniendo la fuerza del PSOE a la de IU, la tendencia dominante sigue siendo de izquierda.

D) Es necesario señalar también los problemas censales que han existido en esta convocatoria electoral y que han impedido ejercer su derecho a numerosos ciudadanos; problemas que, aunque sean solamente técnicos, generan un ambiente de recelo hacia el Gobierno, de quien dependen las oficinas que elaboran aquél. Además, y como dato asombroso, hay que resaltar la decisión de varias Juntas Electorales Provinciales de retrasar el cierre de algunos colegios dos horas después de la oficial (Sevilla, Almeria, Granada, Las Palmas...), a fin de permitir votar a quienes a lo largo del día habian logrado arreglar su situación irregular; ya anunciaban los medios de comunicación los datos de los primeros sondeos cuando estas personas se acercaban a las urnas ${ }^{38}$.

E) Los resultados electorales van a generar, como ya se ha dicho, un nuevo lenguaje político que cambie arrogancia por negociación y pacto ${ }^{39}$, a fin de proporcionar a la mayoría minoritaria, ganadora de las elecciones, los votos necesarios; si bien esto es habitual en Municipios y Autonomías, no lo es hasta ahora en el Parlamento del Estado.

Aunque el electorado socialista manifestó sus preferencias mayoritarias por un pacto con IU, que obligase a llevar a cabo una política más de izquierdas, parece que Felipe González se inclina por un Gobierno minoritario del PSOE (rechazado por todas las partes implicadas un Gobierno de coalición), apoyado por los votos de los nacionalistas $\mathrm{CiU}, \mathrm{CC}$ y PNV para lograr su investidura primero y para aprobar los Presupuestos después, en esta primera fase que estamos analizando. Sobre ello hay que decir lo siguiente.

En primer lugar, detrás de cada pacto siempre queda el interrogante de cuál ha sido el precio de la negociación; en segundo lugar, es más lógico el pacto habitual con determinadas formaciones políticas que un pacto individualizado para cada caso, con partidos distintos e incluso

39 "El escándalo del censo", en Diario 16, 9-VI-93. "La vergüenza de un censo chapucero y manipulable", en EIMundo.

39 J. Pradera, "Bodas y noviazgos", El Mundo, 13-VI-93. J. Lacalle, "Aznar y el pasado de la derecha", Diario 16, 8-VI-93. P. Calvo Hernando, "Un diseño de M. Roca", El Mundo, 7-VI-93. 
antagónicos, que generaría una gran incoherencia política. En tercer lugar, eliminado un pacto con el PP, imposible en una situación ordinaria, es más eficaz la negociación con los nacionalismos que con la opción de Anguita, por lo siguiente:

- No sería bien recibido por los restantes miembros de la Unión Europea, por razones obvias.

- Podría propiciar la unión de PP y nacionalismos, lo que generaria una oposición aún más peligrosa para el Gobierno en minoría; sin embargo, lo que sí parece imposible es que el PP se una a IU, incluso frente al Gobierno.

- Por último, y no por olvidado por todos los comentaristas políticos, menos relevante, IU no ha logrado ningún escaño en el Senado, teniendo solamente los dos representantes designados por las CC.AA.; es decir, el pacto PSOE-IU no sería suficiente para lograr en esta Cámara el número de votos suficiente, poniendo al Gobierno en la necesidad de utilizar habitualmente la excepcionalidad del art. 90 de la Constitución, para salvar los posibles vetos a los proyectos de ley ya aprobados por el Congreso, lo que no parece muy aconsejable politicamente por disfuncional.

F) Finalmente, los analistas políticos internacionales manifiestan su asombro por el triunfo socialista contra todo pronóstico ${ }^{40}$, destacando la personal victoria de Felipe González en una situación económica muy difícil ${ }^{41}$ y la eficacia del "voto del miedo".

40 II Giornale, Le Monde, The Guardian, 7-VI-93.

4T The Times, La Stampa, Libération, La Libre Belgique, 7-VI-93.

42 The Times, The Guardian, 7-VI-93. 